\title{
Prevalência de sobrepeso e obesidade em idosas frequentadoras da Universidade Aberta da Terceira Idade - UATI
}

\author{
Prevalence of overweight and obesity in elderly people attending the Open University \\ of the Elderly-UATI
}

Elisa Leal Aires Pimente ${ }^{1 *}$, Jocyel de Brito Oliveira ${ }^{1}$, Aline dos Anjos Santana ${ }^{2}$, Lorena Maia ${ }^{3}$, Helton

Estrela Ramos ${ }^{4}$

\begin{abstract}
${ }^{1}$ Mestranda(o) do Programa de Pós-Graduação em Processos Interativos de Orgãos e Sistemas (PPGIOS). Universidade Federal da Bahia (UFBA); ${ }^{2}$ Graduada em Nutrição pela Universade dade Federal do Recôncavo da Bahia (UFRB); ${ }^{3}$ Acadêmica do Curso de Medicina. Faculdade de Medicina. UFBA; ${ }^{4}$ Professor Associado. Chefe do Laboratório de Estudo da Tireóide do Instituto de Ciências da Saúde (ICS)/UFBA.
\end{abstract}

\begin{abstract}
Resumo
Introdução: este artigo trata-se da prevalência de sobrepeso e obesidade em idosas frequentadoras da Universidade Aberta da Terceira Idade. Objetivo: avaliar as prevalências de sobrepeso e de obesidade associadas a fatores socioeconômicos e presença de comorbidades, em uma amostra de idosas não institucionalizadas de Salvador-BA, Brasil. Metodologia: um estudo transversal com uma amostra de 72 idosas com mais de 60 anos frequentadoras da Universidade Aberta da Terceira Idade (UATI) vinculada a Universidade Estadual da Bahia (UNEB). Foram aplicados questionários as participantes sobre aspectos pessoais, sócio-demográficos e presença de comorbidades. Resultados: os dados antropométricos avaliados foram o Índice de Massa Corporal (IMC) e da Circunferência da Cintura (CC). Associações entre as variáveis categóricas foram testadas utilizando o teste qui-quadrado com um nível de significância $5 \%$. Observou-se que prevalência de sobrepeso e obesidade de acordo com o IMC foi de $34,48 \%$ e $24,14 \%$, respectivamente e $86,54 \%$ dos idosos apresentaram um substancial aumento da circunferência abdominal. Verificou-se que $13,46 \%$ dos indivíduos eram diabéticos, $63,46 \%$ hipertensos. Conclusão: neste estudo não houve associação com significância estatística entre excesso de peso e renda ou presença de comorbidades (Diabete Melito e Hipertensão).
\end{abstract}

Palavra-Chave: Obesidade. Sobrepeso. Idoso

\begin{abstract}
Introduction: the aim of this study was to evaluate the prevalence of overweight and obesity associated with socioeconomic factors and the presence of comorbidities, in a sample of non-institutionalized elderly women from Salvador-BA, Brazil. Metodology: crosssectional study was carried out with a sample of 72 elderly women over 60 years of age attending the Open University of the Third Age (UATI) linked to the State University of Bahia (UNEB). Questionnaires were applied to participants on personal, socio-demographic and presence of comorbidities. Results: the anthropometric data evaluated were the body mass index (BMI) and waist circumference (WC). Associations between categorical variables were tested using the chi-square test with a $5 \%$ significance level. It was observed that the prevalence of overweight and obesity according to the BMI was high (34.48\% and $24.14 \%$, respectively) and $86.54 \%$ of the elderly showed a substantial increase in waist circumference. It was found that $13.46 \%$ of the individuals were diabetic, $63.46 \%$ were hypertensive. Conclusion: It was observed that BMI was not significantly associated $(p<0.05)$ with income or the presence of comorbidities (Diabetes Mellitus and Hypertension).
\end{abstract}

Keywords: Obesity. Overweight. Old man.

\section{INTRODUÇÃO}

A obesidade é uma doença crônica complexa em que a gordura corporal anormal ou excessiva (adiposidade) prejudica a saúde, aumenta riscos de complicações médicas a longo prazo e reduz expectativa de vida (SEAN et al., 2020).

Sabe-se que o diagnóstico de obesidade é feito a partir do cálculo do índice de massa corporal (IMC; peso / altura 2). Sua presença é definida por um IMC superior a $30 \mathrm{~kg} / \mathrm{m} 2$ e subclassificada em classe 1 (IMC entre 30 e 34,9), classe

Correspondente/Corresponding: *Elisa Leal Aires - Departamento de Biorregulação, Instituto de Saúde e Ciências, Universidade Federal da Bahia, Salvador, Brasil. - Tel: +55 (71) 2137-8880 - E-mail: eairesacademia@yahoo.com
2 (IMC entre 35 e 39,9$)$ e classe 3 (IMC maior ou igual a 40). A circunferência da cintura (CC) está associada de maneira independente ao aumento do risco cardiovascular. A integração do IMC e da CC na avaliação clínica proporciona melhor identificação de indivíduos com maior risco de obesidade, principalmente naqueles com IMC mais baixo (SEAN et al., 2020).

Associação Brasileira para o Estudo da Obesidade e da Síndrome Metabólica divulgou dados da Organização Mundial de Saúde no qual estima que em 2025 cerca de 2,3 bilhões de adultos no mundo estejarão acima do peso, dos quais 700 milhões de indivíduos com obesidade. No Brasil, essa doença crônica aumentou mais de $65 \%$ nos 
últimos anos, saindo de 11,8\% em 2006 para 19,8\% em 2018 (ABESO, 2009).

De acordo com a pesquisa da Vigilância de Fatores de Risco e Proteção para Doenças Crônicas por Inquérito Telefônico realizada em 2019 realizada pelo Ministério da Saúde, a prevalência de obesidade nas capitais está estimada em $20,3 \%$, sendo semelhante entre homens e mulheres. A frequência de obesidade aumentou com a idade até os 64 anos, para homens, e até os 54 anos, para mulheres. A presença de excesso de peso nas capitais brasileiras é de $55,4 \%$, sendo discretamente maior entre homens $(57,1 \%)$ do que entre mulheres $(53,9 \%)(8)$. Estes dados chamam a atenção para a epidemia global de obesidade e a necessidade da atuação em prevenção primária do excesso de peso (BRASIL, 2020).

As modificações ocorridas no cenário demográfico proporcionaram um aumento substancial do número de pessoas idosas que se sobrepõe a uma maior prevalência de obesidade. Dessa forma, o predomínio de doenças crônicas, complexas e onerosas na população idosa sofre impacto negativo na presença da obesidade. A inflamação crônica de baixo grau associada ao excesso de massa gorda promove resistência a insulina, perda de massa muscular, contribui com desfechos desfavoráveis de doenças crônicas aumentando custos com a saúde (MARQUES et al., 2005).

$\mathrm{Na}$ idade avançada, a composição corporal muda a medida que a massa gorda aumenta e se redistribui (JAVED et al., 2020). A massa livre de gordura composta principalmente por músculo esquelético diminui em $40 \%$ entre as idades de 20 e 70 anos (VILAREALL et al., 2005). Após 70 anos, tanto a massa gorda quanto a massa magra diminuem juntas. Com a redistribuição da massa gorda, ocorre um aumento do depósito de gordura visceral e muscular. A gordura ectópica está associada a um painel desfavorável de adipocinas e maior secreção de citocinas pro-inflamatórias contribuindo para o estado inflamatório (SCHRAGER et al., 2007).

O balanço energético é o fator determinante da massa gorda. No idoso, a ingestão alimentar está mantida ou até reduzida, portanto, a diminuição do gasto energético desempenha um papel mais preponderante no aumento da massa gorda associada ao envelhecimento.

Obesidade sarcopênica é a associação do excesso de adiposidade com redução da massa muscular (CRUZ-JENTOFT, et al., 2019). Essa condição clínica é mais reportada em idosos devido a perda de massa muscular progressiva associada ao envelhecimento. A obesidade exacerba a sarcopenia, aumenta a infiltração gordurosa do tecido muscular, piora performance física e aumenta risco de mortalidade. Uma preocupação com o tratamento da obesidade nessa população idosa é a perda de massa muscular associada a perda ponderal e agravamento da sarcopenia (MCKEE et al., 2018).

Devido a elevação na expectativa de vida e maior prevalência de obesidade no mundo, o presente estudo foi realizado objetivando determinar a prevalência de obe- sidade na população idosa avaliada e sua associação com variáveis sócio-demográficas e presença de comorbidades.

\section{METODOLOGIA}

Este estudo observacional, analítico e transversal faz parte de um projeto de estudo que avalia a associação entre sarcopenia e função tireoidiana em idosas não institucionalizados, realizado nos anos de 2018 e 2019. A amostra foi composta por idosas com mais de 60 anos, frequentadoras da Universidade Aberta da Terceira Idade (UATI) da Universidade Estadual da Bahia (UNEB), na cidade de Salvador. As voluntárias foram convidadas a participar do estudo no local das suas aulas regulares.

O projeto com o CAAE 93016218.9.0000.5662 foi aprovado pelo Comitê de Ética do Instituto de Ciências da Saúde (ICS) e todas as voluntárias assinaram o termo de consentimento livre e esclarecido (TCLE) após orientação quanto aos objetivos, procedimento operacionais e garantia de preservação da identidade.

As entrevistas foram realizadas na própria instituição, antes das atividades nas classes. Foram aplicados questionários sobre condições socioeconômicas, presença de comorbidades, hábitos de vida e nutrição. Foram incluídas no estudo 72 idosas frequentadoras da UATI com idade superior a 60 anos que consentiram e assinaram o TCLE. As participantes que não preencheram a totalidade dos questionários ou não fizeram a avaliação antropométrica foram excluídas. Foram incluídos 72 participantes do sexo feminino com mais de 60 anos, após exclusão de 20 voluntárias devido a presença de dados incompletos, permaneceram no estudo 52. A mediana da idade foi 70 anos (IIQ =65; 73), variando de 60 a 95 anos.

A avaliação antropométrica, com mensuração da circunferência da cintura (CC), peso, altura e cálculo do IMC foi realizada após aplicação dos questionários. $O$ peso foi medido em quilograma utilizando-se uma balança digital portátil da marca Plenna modelo Sport, com capacidade para $150 \mathrm{~kg}$ e divisão em gramas. Os voluntários foram pesados trajando roupas leves e descalços. A altura foi mensurada em metros, com o voluntário em posição ereta e olhar fixo na altura do horizonte, com os pés descalços, unidos, no chão e encostados na parede utilizando um estadiómetro portátil marca Sanny, com capacidade máxima de $204 \mathrm{~cm}$ e precisão de $5 \mathrm{~mm}$. A CC foi aferida com uma fita métrica inelástica com precisão de $1 \mathrm{~mm}$ posicionada na altura do umbigo.

As varíaveis sócio-demográficas avaliadas foram: idade em anos, renda, grau de escolaridade e presença de comorbidades crônicas. Inicialmente, fez-se uma análise descritiva dos dados através do cálculo de medidas de tendência central (mediana) e de dispersão (intervalo interquartílico) para as variáveis de natureza quantitativa e frequências relativas.

Para avaliar a associação entre variáveis qualitativas utilizou-se o Exato de Fisher, uma vez que as suposições necessárias para o uso do teste Qui-Quadrado não foram 
satisfeitas. Já para avaliar a diferença na mediana da renda segundo as categorias de IMC e de CC utilizou-se o teste de Wilcoxon-Mann-Whitney para dados independentes. Optou-se por tal método pelo fato de não ter sido observado um comportamento similar ao da distribuição normal para a variável renda. Utilizou-se um nível de significância de 5\% para interpretação dos resultados.

\section{RESULTADOS}

Esse estudo é composto por 72 participantes do sexo feminino com mais de 60 anos, sendo a mediana de idade 70 anos (IIQ $=65 ; 73)$, variando de 60 a 95 anos. A renda individual média foi de $\mathrm{R} \$ 1.810,509$ ( $\mathrm{DP}=\mathrm{R} \$ 1.087,961)$, a sua mediana foi de $\mathrm{R} \$ 1.751,00$ (IIQ $=875,15,2.624,188)$, variando de $R \$ 816,00$ a $R \$ 5244,75$. Em relação ao grau de escolaridade, $9,62 \%$ da amostra tinham o estudo fundamental, $55,77 \%$ o ensino médio, $26,92 \%$ o ensino superior e $7,69 \%$ pós graduação.

A mediana do IMC foi de $26,01 \mathrm{~kg} / \mathrm{m}^{2}$ (IIQ 23,93, $29,58)$, variando de 21,18 a 34,80 . A mediana da CC foi de $90,55 \mathrm{~cm}$ (IIQ 83,75; 97,5), variando de $68 \mathrm{~cm}$ a $112 \mathrm{~cm}$. As prevalências de sobrepeso e obesidade com base no IMC foram de $34,48 \%$ e $24,14 \%$, respectivamente. Nenhuma das voluntárias tinha baixo peso. Maior risco cardiovascular associado a circunferência da cintura foi encontrado em $86,54 \%$ das participantes. $63,46 \%$ delas tinham Hipertensão Arterial e 13,45\% Diabete Melito.

A amostra foi composta por $44,23 \%$ de voluntários com peso normal e $55,77 \%$ com excesso de peso. Entre os voluntários com excesso de peso (sobrepeso ou obesidade), $17,24 \%$ tinham o ensino fundamental, $55,17 \%$ o ensino médio, $17,24 \%$ o ensino superior e $10,34 \%$ o pós-graduação. A presença de Diabete Melito e Hipertensão Arterial foi de $13,04 \%$ e $60,87 \%$ naquelas com peso normal e $13,79 \%$ e $65,52 \%$ com excesso de peso, nessa ordem. A frequência de Hipertensão Arterial foi maior entre o grupo de maior CC e maior risco cardiovascular, $71,11 \%$. Não houve diferença significativa na presença de Diabete Melito entre os grupos com CC normal e elevada.

Não foram observadas no estudo associações com significância estatística entre excesso de peso e comorbidades (Diabete Melito e HAS). Entre elevação da CC e Hipertensão Arterial foi encontrada associação com significância estatística. Não houve evidência de associação entre grau de escolaridade e excesso de peso. Também não foi observada diferença estatisticamente significante entre os grupos de peso normal e excesso de peso em relação a mediana da renda.

Tabela 1 - Características da amostra

\begin{tabular}{l|r|c}
\hline \multicolumn{1}{c|}{$\mathbf{N}$} & $\mathbf{5 2}$ & $\begin{array}{c}\mathbf{1 0 0 \%} \text { sexo } \\
\text { feminino }\end{array}$ \\
\hline Idade & 70 anos $(65 ; 73)$ & 60 a 95 \\
\hline Peso Normal & 23 & $44,23 \%$ \\
\hline Sobrepeso & 18 & $34,62 \%$ \\
\hline Obesidade & 11 & $21,15 \%$ \\
\hline Excesso de peso & 29 & $55,77 \%$ \\
\hline Circunferência Cintura & $90,55 \mathrm{~cm}(83,75 ; 97,5)$ & \\
\hline Diabete Melito & 7 participantes & $13,46 \%$ \\
\hline
\end{tabular}

\begin{tabular}{l|l|l}
\hline Hipertensão Arterial & 33 participantes & $63,46 \%$ \\
\hline
\end{tabular}

Fonte: Autoria própria

Tabela 2 - Associação entre IMC e Diabete Melito

\begin{tabular}{l|r|r|r}
\hline $\begin{array}{c}\text { Diabete } \\
\text { Melito }\end{array}$ & Peso Normal & $\begin{array}{r}\text { Excesso de } \\
\text { Peso }\end{array}$ & Total \\
\hline Não & 20 & 25 & 45 \\
& 44,44 & 55,56 & 100,00 \\
& 86,98 & 86,21 & 86,54 \\
\hline Sim & 3 & 4 & 7 \\
& 42,86 & 57,14 & 100,00 \\
& 13,04 & 13,79 & 13,46 \\
\hline Total & 23 & 29 & 52 \\
& 44,23 & 55,77 & 100,00 \\
& 100,00 & 100,00 & 100,00 \\
\hline
\end{tabular}

Fisher's exact $=1.00$ (sem associação)

Fonte: Autoria própria

Tabela 3 - Associação entre IMC e hipertensão arterial

\begin{tabular}{l|r|r|r}
\hline $\begin{array}{c}\text { Hipertensão } \\
\text { Arterial }\end{array}$ & Peso Normal & $\begin{array}{r}\text { Excesso de } \\
\text { Peso }\end{array}$ & Total \\
\hline Não & 9 & 10 & 19 \\
& 47,37 & 52,63 & 100,00 \\
& 39,13 & 34,48 & 36,54 \\
\hline Sim & 14 & 19 & 33 \\
& 42,42 & 57,58 & 100,00 \\
& 60,87 & 65,52 & 63,46 \\
\hline Total & 23 & 29 & 52 \\
& 44,23 & 55,77 & 100,00 \\
& 100,00 & 100,00 & 100,00 \\
\hline
\end{tabular}

Fisher's exact $=0,778$ (sem associação)

Fonte: Autoria própria

Tabela 4 - Associação entre CC e hipertensão arterial

\begin{tabular}{l|r|r|r}
\hline $\begin{array}{c}\text { Hipertensão } \\
\text { Arterial }\end{array}$ & Peso Normal & $\begin{array}{r}\text { Excesso de } \\
\text { Peso }\end{array}$ & Total \\
\hline Não & 6 & 13 & 19 \\
& 31,58 & 68,42 & 100,00 \\
Sim & 15,71 & 28,89 & 36,54 \\
\hline Total & 3,03 & 32 & 33 \\
& 14,29 & 96,97 & 100,00 \\
& 7 & 71,11 & 63,46 \\
\hline
\end{tabular}

Fisher's exact $=0,007$ (evidência de associação)

Fonte: autoria própria

\section{DISCUSSÃO}

Conforme os dados do Ministério da Saúde, no Brasil, a ocorrência de sobrepeso e obesidade aumenta durante a vida adulta e reduz após os 60 anos. Segundo dados do Vigitel (BRASIL, 2020), entre homens, a frequência do excesso de peso aumentou com a idade até os 44 anos e entre as mulheres aumentou com a idade até os 64 anos.

A prevalência de excesso de peso no conjunto de 
população adulta das principais capitais do Brasil foi de $55,4 \%$, com discreta diferença entre os sexos. Estudos na população dos Estados Unidos apontam uma prevalência geral de obesidade ajustada por idade de $35 \%$ entre homens e $40,4 \%$ entre mulheres, taxas paralelas às observadas na população geriátrica, $37,1 \%$ e $33,6 \%$ entre homens e mulheres, respectivamente (MALENFANT et al., 2019). Nesse estudo, foi encontrada uma prevalência de excesso de peso de $55,7 \%$, de sobrepeso $34 \%$ e $21,15 \%$ para obesidade em idosas com mais de 60 anos. Não foi possível fazer uma análise comparativa entre os sexos devido a amostra do estudo ser do sexo feminino (BRASIL, 2020).

O processo de envelhecimento está associado a perda progressiva da massa muscular e óssea e acúmulo de massa gorda, com piora da composição corporal (SANTANASTO et al., 2017). Este processo envolve alterações fisiológicas e nutricionais que se manifestam pela perda de altura, peso e massa muscular e aumento da massa gorda, incluindo redistribuição de tecido adiposo, com acúmulo de gordura no tronco e vísceras. Essas alterações associadas a idade resultarão em má regulação energética, redução dos níveis hormonais e alterações na taxa metabólica impactando na saúde do idoso (FUSIANA et al., 2016).

A população idosa apresenta maior prevalência de doenças crônicas não infeciosas, como Diabete Melito e Hipertensão arterial. A inflamação sistémica crônica de baixo grau associada ao acúmulo e distribuição ectópica de massa gorda pode contribuir de maneira negativa com o desfecho dessas patologias. No estudo atual, a prevalência de Diabete e HAS foi de $13,46 \%$ e $63,46 \%$ respectivamente.

A redução do IMC no idoso as custas da perda de massa muscular associada ao aumento da CC leva aos riscos clínicos da obesidade que aparecem em um IMC mais baixo em idosos (FUSIANA et al., 2016). No presente estudo, não foi encontrado baixo peso entre as participantes.

O aumento da CC está associado a resistência a insulina, inflamação sistêmica crônica e risco cardiovascular elevado (SEAN et al., 2020). Foi encontrada associação com significância estatística entre Hipertensão Arterial e elevação da CC no estudo atual.

\section{CONCLUSÃO}

A prevalência de excesso de peso na amostra avaliada foi de $58,62 \%$ estando próximo ao valor encontrado na população adulta estimada pelo Vigitel (BRASIL, 2020). Não foi encontrado associação estatística entre excesso de peso e as comorbidades Diabete Melito e Hipertensão. A ausência de associação provavelmente é em consequência do tamanho da amostra.

O panorama geral da população está mudando e mostrando uma prevalência maior de obesidade e aumento da expectativa de vida. Essas mudanças impactam diretamente em questões sociodemográficas e custos com a saúde. A forma como a população envelhece, a atenção dada na promoção a saúde e controle de doenças crônicas, merecem receber atenção da comunidade científica e órgão governamentais.

\section{REFERÊNCIAS}

ASSOCIAÇÃO BRASILEIRA PARA O ESTUDO DA OBESIDADE E DA SÍNDROME METABÓLICA (ABESO). Mapa da obesidade. 2009. Disponível em: https://abeso.org.br/obesidade-e-sindrome-metabolica/mapa-daobesidade/. Acesso em: 20 nov. 2020

BRASIL. Ministério da Saúde. Secretaria de Vigilância em Saúde. Departamento de Análise em Saúde e Vigilância de Doenças Não Transmissíveis. Vigitel Brasil 2019: vigilância de fatores de risco e proteção para doenças crônicas por inquérito telefônico: estimativas sobre frequência e distribuição sociodemográfica de fatores de risco e proteção para doenças crônicas nas capitais dos 26 estados brasileiros e no Distrito Federal em 2019. Brasília, 2020. 137p.

CRUZ-JENTOFT, A. J. et al. Sarcopenia: revised European consensus on definition and diagnosis. Age ageing, London, v.48, n.1, p.16-31, 2019

JAVED, A. A. et al. Body mass index and all-cause mortality in older adults: A scoping review of observational studies. Obes rev., Oxford, v. 21, n. 8, e13035, 2020

MALENFANT, J. H. et al. Obesity in geriatric population - a global health perspective. J. Global Health Reports, United Kingdom, v.3, p. e2019045, 2019

MARQUES, A P. de O. et al. Prevalência de obesidade e fatores associados em mulheres idosas. Arq. bras. endocrinol. metab., São Paulo, v. 49, n. 3, p. 441-448, 2005

MCKEE, A. M.D. et al. Obesity in the Elderly. In: Endotext. South Dartmouth (MA), 2018.

SANTANASTO, A. J. et al. Body Composition Remodeling and Mortality: The Health Aging and Body Composition Study. J. gerontol. A, Biol, sci, med. sci., Washington, v.72, n.4, p.513-519, 2017.

SCHRAGER, M. A. et al. Sarcopenic obesity and inflammation in the InCHIANTI study. J. appl. physiol., Washington, v.102, n.3, p. 919-925, 2007

VILAREALL, D. T. et al. Obesity in older adults: technical review and position statement of the American Society for Nutrition and NAASO, The Obesity Society. Am. j. clin. nutr., Bethesda, v.82, n.5, p.923-934, 2005.

WHARTON, S. et al. Obesity in adults: a clinical practice guideline. CMAJ, Ottawa, v.192, n. 31, p. E875-E891, 2020.

Submetido em: 07/12/2020

Aceito em: 14/12/2020 